\title{
Spatial-spectral flexible optical networking: enabling switching solutions for a simplified and efficient SDM network platform
}

\author{
I. Tomkos ${ }^{* a}$, P. Zakynthinos ${ }^{\mathrm{a}}$, D. Klonidis ${ }^{\mathrm{a}}$, \\ D. Marom ${ }^{\mathrm{b}}$, \\ S. Sygletos ${ }^{\mathrm{c}}, \mathrm{A}$. Ellis $^{\mathrm{c}}$, \\ E. Salvadori ${ }^{\mathrm{d}}$, D. Siracusa ${ }^{\mathrm{d}}$, \\ M. Angelou ' G. Papastergiou', \\ N. Psaila ${ }^{f}$ \\ J. F. Ferran ${ }^{\text {g, }}$ \\ S. Ben-Ezra ${ }^{\text {h, }}$ \\ F. Jimenez ${ }^{i}$, J.P. Fernández-Palacios ${ }^{i}$ \\ ${ }^{a}$ Athens Information Technology, Greece; \\ ${ }^{\mathrm{b}}$ The Hebrew University of Jerusalem, Israel \\ ${ }^{\mathrm{c}}$ Aston University, UK \\ ${ }^{\mathrm{d}}$ CreateNet, Italy

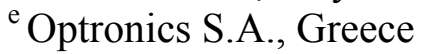 \\ ${ }^{\mathrm{f}}$ Optoscribe Ltd., UK \\ ${ }^{\mathrm{g}} \mathrm{W}$-onesys S.L., Spain \\ ${ }^{\text {h }}$ Finisar Israel Ltd., Israel \\ ${ }^{i}$ Telefonica Investigacion y Desarrollo S.A., Spain
}

\begin{abstract}
The traffic carried by core optical networks grows at a steady but remarkable pace of 30-40\% year-over-year. Optical transmissions and networking advancements continue to satisfy the traffic requirements by delivering the content over the network infrastructure in a cost and energy efficient manner. Such core optical networks serve the information traffic demands in a dynamic way, in response to requirements for shifting of traffics demands, both temporally (day/night) and spatially (business district/residential). However as we are approaching fundamental spectral efficiency limits of singlemode fibers, the scientific community is pursuing recently the development of an innovative, all-optical network architecture introducing the spatial degree of freedom when designing/operating future transport networks. Spacedivision-multiplexing through the use of bundled single mode fibers, and/or multi-core fibers and/or few-mode fibers can offer up to 100-fold capacity increase in future optical networks. The EU INSPACE project is working on the development of a complete spatial-spectral flexible optical networking solution, offering the network ultra-high capacity, flexibility and energy efficiency required to meet the challenges of delivering exponentially growing traffic demands in the internet over the next twenty years. In this paper we will present the motivation and main research activities of the INSPACE consortium towards the realization of the overall project solution.
\end{abstract}

Keywords: Space Division Multiplexing, Flexible Optical Networking

*itom@ait.gr; phone +30 210668 2771; fax +30 210668 2729; www.ait.gr

Next-Generation Optical Communication: Components, Sub-Systems, and Systems III, edited by Guifang Li, Proc. of SPIE Vol. 9009, 90090H · @ 2014 SPIE · CCC code: 0277-786X/14/\$18 · doi: 10.1117/12.2045323 


\section{INTRODUCTION}

Over the last years, network traffic has consistently grown at an exponential rate, and there is no indication this relentless trend will cease. At present, industry is hard-pressed to identify how future networks will continue to scale in capacity, energy consumption, and economic viability as present day technologies are being stretched to their limits. The nascent technology of space-domain multiplexing (SDM) for high capacity transmission is the only solution with the scaling potential to meet future demands [1]-[3]. However, there is still a large technological chasm between the transport solution and the SDM network implementation.

A large number of significant innovations that are able to offer a capacity increase in fiber systems practically by a factor of around 10-20 (compared to legacy WDM systems at $10 \mathrm{~Gb} / \mathrm{s}$ and $100 \mathrm{GHz}$ spacing) have emerged over the last couple of years. However the capacity increase efforts targeted purely the frequency and the signal encoding domains, in an effort to increase the spectral density in fiber links [1]. This led to the definition of the spectral super-channel approach that utilizes spectrally flexible (a.k.a. elastic) formats for the dynamic and adaptive allocation of end-to-end demands with variable contents (i.e. requested data rates). However, although the spectral super-channel approach can optimize the network resources by offering increased spectral utilization, it has a limited growth potential due to the "capacity crunch" [4], on account of the finite transport capacity of a given single mode fiber core and the limited gain bandwidth of optical amplifiers [5]. These issues will eventually lead to scalability issues and blocking situations for evolving spectral super-channels based optical networks [6].

The only other evident solution to extend the capacity of optical communication systems relies on the use of the space domain. The simplest way to achieve this is to deploy multiple systems in parallel. However, by simply increasing the number of systems, the cost and power consumption also increases linearly. In order to limit the increase in cost and power consumption, a degree of sharing has to be introduced, in terms of elements and resources among the spatially multiplexed systems that are deployed in parallel; in other words this denoted the need of a "spatial integration of system elements" [1]. To this extend significant research efforts have focused on the development and performance evaluation of few-mode fibers (FMF) and multi-core fibers (MCF) (e.g.[2], [3]), which can be seen as 'integrated fiber' media. This work is further supported by the development of integrated optical amplification systems as for example in [7], as well as the significant development efforts in the field of $\mathrm{Tb} / \mathrm{s}$ integrated transponders (nicely summarized in [8]).

The potential capacity increase offered by the exploitation of the space domain can be an important step in tackling the continuously growing traffic demand patterns seen by network operators. However, the current spatial integrating solutions are targeting purely the capacity increase in point-to-point transmission systems. What still remains unexplored is the introduction of the space dimension in optical networking. This in turn requires additional technology developments in the field of spatial demultiplexing and most importantly in the switching of the optical contents over both the space and spectrum dimensions. The introduction of a multi-dimensional (spectral and spatial) optical networking is envisioned to offer an additional degree of freedom in terms of the overall resource optimization of the system.

This paper addresses the scientific approaches that are proposed and followed by the EU funded project INSPACE, providing a novel networking approach that extends the established spectral flexibility concepts to the SDM domain. The networking approach in combination with the proposed technology developments in spatial signal manipulation and switching is expected to significantly simplify the super-channel allocation and control mechanisms, by removing current limitations related with the wavelength continuity and fragmentation issues. The new concept utilizes the benefits of the high capacity, next generation, few-mode/multi-core fiber infrastructures, providing also a practical short term solution, since it is directly applicable over the currently installed multi-fiber cable links. The realization of INSPACE approach is enabled by the development of novel multi-dimensional spatial-spectral switching nodes, which are fabricated by extending the designs of the existing flexible WSS nodes, incorporating advance mode/core adapting techniques. The concept is further supported by novel processing techniques that minimize the mode/core interference as well as new network planning algorithms and control plane extensions that are enhanced with the space dimension. 


\section{INTRODUCING THE SDM DOMAIN IN FLEXIBLE OPTICAL NETWORKS}

In the framework of the research activities of EU project INSPACE we introduce a novel logical hierarchical structure for next generation multidimensional dynamic and elastic optical networks, based on enabling switching, transmission and processing technologies that allow the connection between spectral and spatial networking. We focus on:

a) Exploring the capabilities offered by the additional degrees of freedom in signal processing and multiplexing for both multi-mode and multi-core fibre systems, with the purpose to exploit the emerging networking benefits arising from a combined Spatial/Spectral/Signal coding domain.

b) Designing multi-dimensional resource allocation algorithms and study the required control plane extensions with the goal to evaluate the benefits of multi-dimensional signal allocation in terms of resource optimization, energy consumption reductions and system cost savings

c) Developing novel enabling technologies in support of the multi-dimensional networking concept by focusing on node switching hardware adapted to support SDM fiber links (SMF array/FMF/MCF) addressing the spatial and spectral domains

Figure 1 shows an envisioned multidimensional structure for channel allocation where a number of degrees of freedom emerge and each of them can contribute to increasing the total transmission capacity and simplifying the routing of network flows. The proposed logical hierarchal structure considers various fibre link types, with spectrally elastic contents, carrying multi-format signals. The main considered SDM fibre link types are:

- An SMF bundle in a multi-fibre cable.

- A multi-core fibre (MCF) with isolated cores.

- A multi-core fibre (MCF) with closely spaced cores.

- A few-mode fibre (FMF).

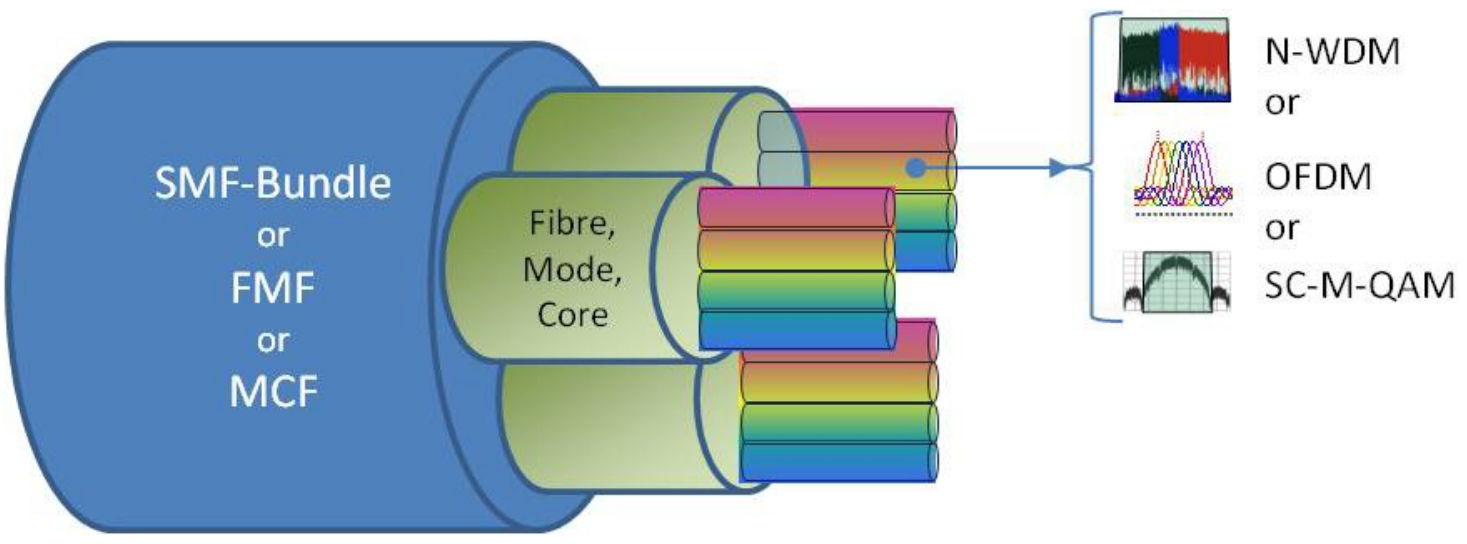

Figure 1 - Multi-dimensional structure for channel allocation, spanning space, wavelength, and time

The aforementioned SDM structures give rise to the following hierarchical levels for networking:

- The spatially multiplexed fibre, core or mode level (at the highest layer)

- The spectrally multiplexed level (at the middle layer) considering either

- fixed grid spectral containers: single slot channels or super-channels based on spectral concatenation of multiple signals each on a dedicated frequency slot.

- flex grid spectral containers: where single optical signals or super channel structures are allocated.

- The signal format level (at the lowest layer) considering m-QAM signals multiplexed together in various ways. 
Figure 2 represents the flexible channel allocation concept where channels can be accommodated over one or multiple modes/cores occupying a single or multiple spectral slots and transmitted using different modulation levels.

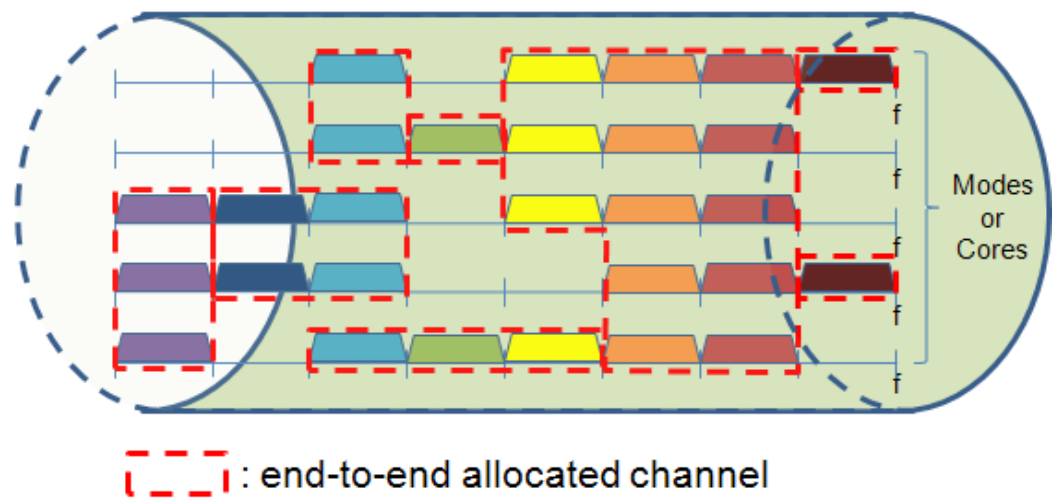

Figure 2 - Multi-dimensional channel allocation concept: Spatial expansion of the spectrum over few modes/multiple cores and therefore definition of a super-channel over two dimensions (instead of the spectrum only dimension) denoted by red dashed lines

To realize multi-dimensional spatial networking, we need however to develop new optical switching nodes with capabilities to switch traffic at different granularity levels. In the following section we highlight the novel relevant development activities within EU project INSPACE.

\section{SPATIAL DEMULTIPLEXING AND MULTI-DIMENSIONAL SWITCHING NODE}

A multi-dimensional switching node may use all three granularity levels (space, wavelength, and time) or a subset of two of them depending on the technology capabilities. Utilizing the time domain requires active components and hence is reserved to IP routers that can utilize burst type communication or TDM leading to significant energy inefficiency. INSPACE will focus on the optical level, where information can be routed transparently and most efficiently. Aiming for ultra-low energy consumption figures, the frequency and space dimensions are explored. We present five possible switch design solutions for a spatial-spectral node design that will serve to demonstrate the concepts behind INSPACE:

a) Bundle of SMF and independent fibre switching with designated WSS/OXC (space-spectrum granularity),

b) MCF decoupling and independent core switching with designated WSS/OXC (space-spectrum granularity),

c) MCF decoupling and WSS joint spectral switching across all cores in space (spectral granularity spanning space),

d) FMF decoupling and WSS joint spectral switching across all modes in space (spectral granularity spanning space),

e) FMF spectral switching of all fibre contents at once (w/o decoupling) with custom WSS (spectral granularity spanning space)

These five scenarios can potentially lead to a requirement for the development of only two novel node solutions. The first offers switching granularity down to the spectral and spatial domains. The second node solution offers granularity at the spectral domain (as is performed today using wavelength-selective switches, WSS), yet performed across all spatial channels simultaneously.

A possible implementation of the spatial-spectral granularity node design is illustrated in Figure 3 (left). This solution is only compatible for switching SDM-WDM channels carried either on an SMF array or MCF where the cores are sufficiently displaced such that the modes do not exhibit any coupling (isolated cores). This switching node design offers the ability to cross connect between several SDM links, by separating the spatial channels and treating each as an 
independent spatial channel. By placing a conventional WSS (single input port, multiple output ports) on every spatial channel, individual spectral channels can be routed to corresponding spatial channels of target SDM link, where they are multiplexed by a second WSS (multiple input ports, single output port). This is identical to the architecture used in cross connecting SMF network links today, but scaled to support multiple independent and parallel spatial conduits by scaling the WSS count. In addition, support for colorless-directionless-contentionless (CDC) add-drop is provided by incorporating an optical cross-connect. If we want to enable spatial channel routing between SDM links and within each SDM group, then we can pack signals bound to a common destination onto a single spatial super-channel, such that at networks nodes downstream the spatial channel need not be spectrally separated and can then be routed as a whole. This action also potentially reduces the number of WSS that are deployed in a node. This scenario is depicted in Figure 3 (left), where paths denoted ' $a$ ' and ' $b$ ' merge non-overlapping spectral channels from two cores of the same SDM link onto one spatial channel. This will allow direct core-to-core routing in subsequent switching nodes (denoted 'c').
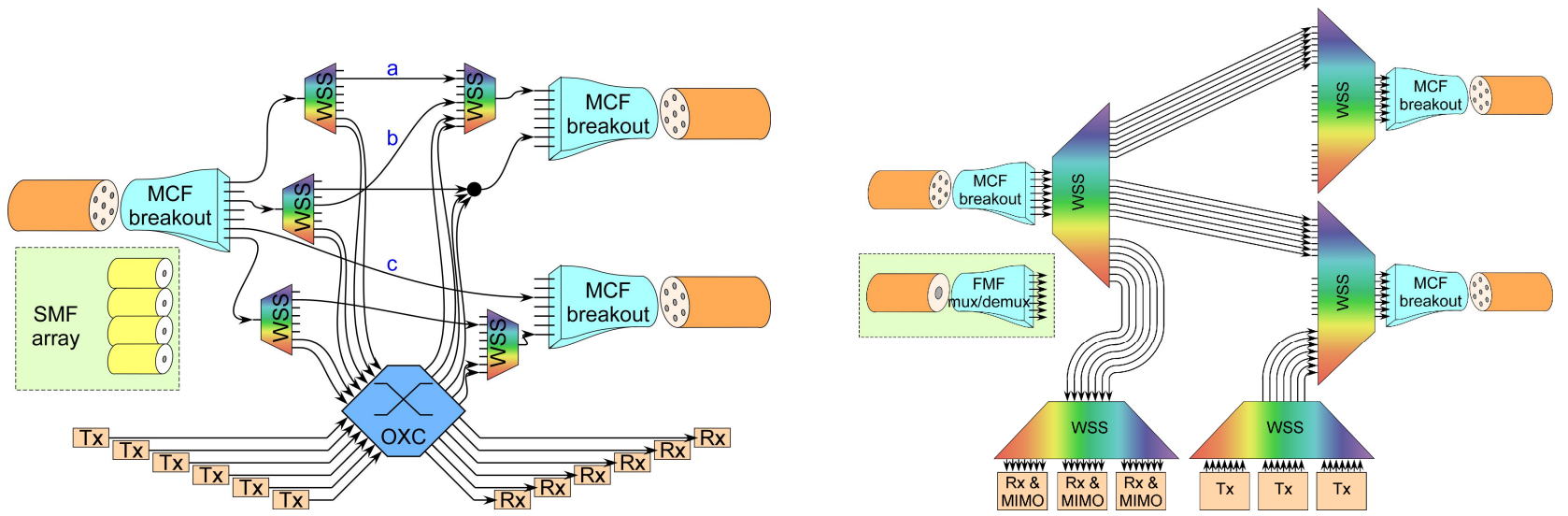

Figure 3 - Left: Possible implementation of a space-spectrum granularity switching solution, gaining access to individual spatial channels, applicable for MCF with isolated cores or SMF array, Right: Implementation of a spectral switching solution, where spatial modes are switched jointly (on a wavelength basis with modified WSS with interfacing spatial demultiplexer

Space-spectrum granularity offers full flexibility in channel allocation. Individual narrow channels $(100 \mathrm{~Gb} / \mathrm{s}) \mathrm{can}$ be assigned to any available spatial/spectral slot using any modulation format and bit rate. Additional parameters affecting channel routing selection are OSNR and nonlinear impairments in available paths to destination, and blocking conditions. Terabit super-channels can be implemented in the spectral domain by occupying multiple spectral slots of a single spatial channel, or in the spatial domain by utilizing a single spectral slot spread out over all the spatial channels. Intermediate solutions, where a super-channel is spread over a subset of spatial and spectral channels are also permissible. Determining which solution is best has to take into account several parameters. Reasons in favor of spatial super-channel result from the following: a single laser can be used both in the transmitter and in the coherent receiver enabling common processing for phase estimation, supported by common flex-grid WSS, spatial channel nonuniformities can be handled by aware transponders and FEC spread spatially. Reasons in favor of spectral super-channels result from the following: spatial channels may be more prone to tight optical filtering (in spectral domain the entire spectrum is contiguous provisioned in flex grid WSS), synchronization of signal tributaries maintained, network links need not have matched degree of parallelism.

An implementation of the spectral granularity applied across all spatial conduits is illustrated in Figure 3 (right). Since this solution keeps the entire spatially parallel channels intact, it can support SDM links where cross-coupling occurs, such as closely packed cores of a MCF and FMF. Note that SDM links with no cross-coupling can also adopt this solution as it requires significantly less hardware, but will lose the provisioning spatial degree of freedom. The switching node design is basically again the same cross-connecting solution implemented with WSS, however this time the WSS are specialized in that they offer the same single input multiple, output routing functionality, but applied to all spatial channels simultaneously. The architecture shown has the ingress side WSS distribute WDM channels to one of multiple egress side WSS's, and one drop WSS that further separates wavelength channels for detection by a receiver array and MIMO processing. This simple solution is colorless and contention-less, but is directional (deployed transmitters and receivers are assigned to a SDM link). The simplicity of the switching architecture makes it as appealing as the SMF WSS in today's networks. The customized WSS required for joint switching of parallel channels may support MCF and 
FMF with spatial demultiplexers. A different WSS variant that supports spectral switching for all spatial modes is by a WSS operating on directly with FMF at its I/O interfaces. However, this FMF WSS has different spectral switching characteristics that have to be taken into account in network design and operation.

The advantages of the simplified hardware, spectral granularity switching node design further extend to the network provisioning controls. The spatial parallelism provides a capacity boost, while management remains at the wavelength level, hence most existing wavelength reconfigurable switching protocols can be carried over. Since the spatial parallelism benefit cannot be removed, it may imply that the finest bandwidth granularity to be switched may be less than $50 \mathrm{GHz}$, as high capacity spatial super-channels are always formed but not necessarily required. If fixed grid WSS are employed for spatial super-channel formation, then spectrum defragmentation issues plaguing spectral superchannels are eliminated.

\section{ROUTING ALGORITHMS FOR OPTIMIZATION OF SDM NETWORKS}

During the network planning procedure, resources - such as transponders, spectrum and spatial cores/modes- need to appropriately provisioned to connections in order to satisfy the defined optimization objectives. The optimization objective can be tuned to meet different criteria - including the minimization of the required spectral resources, the minimization of the capital expenditures for network equipment, and the maximization of the energy efficiency. The set of traffic demands that have to be accommodated, and the capabilities that are offered by the considered network equipment are generally considered as input. The network topology can be either given as input or provided as a result from the optimization process.

Conventional fixed-grid networking solutions (as shown in Figure 4 (a)) require the application of Routing and Wavelength Assignment (RWA) algorithms. These algorithms guarantee that the traffic demand is appropriately routed between all source-destination node-pairs. RWA algorithms also impose constraints that are required in the network planning process, such as wavelength continuity (i.e., imposing that the same wavelength is used in all of the links traversed by the connection) and single wavelength assignment (i.e. imposing that on each link each wavelength can be used by only one connection). The emergence of flexible optical networking (as shown in Figure 4 (b)) as a method to increase resource efficiency and provide advanced functionalities poses significant challenges on the networking level. To this end, advanced resource allocation algorithms are developed - as the conventional routing and wavelength assignment (RWA) algorithms of traditional WDM networks can no longer be applied. Instead of assigning a certain wavelength to each connection, a number of contiguous spectrum slots are now to be assigned. Additionally, the continuity of these spectrum slots should be guaranteed in a similar manner as wavelength continuity constraints are imposed. This leads to the development of routing and spectrum allocation algorithms (RSA) [9]. Moreover, as additional degrees of freedom are allowed by flexible optical networks - new relevant constraints are required to be considered. For example as the modulation level can be selected on a connection basis, constraints tying it to the required bit-rate of the traffic demand as well as to the achieved transparent reach are necessary. To this end routing modulation level and spectrum allocation algorithms (RMLSA) have been recently proposed [10,11]. The discussed algorithms can address the offline network planning phase $[9,10]$, or they can be applied to dynamically provision connection requests $[10,12]$. As connections are dynamically established and released, the issue of bandwidth fragmentation arises - leading to increased blocking probabilities. Thus, the development of spectrum defragmentation algorithms is required [13, 14]. As energy efficiency has gained increasing importance due to environmental awareness and the pressure to drive down the operational costs, the potential benefits of flexible optical networks in terms of energy efficiency have been examined [15].

With the advent SDM technologies, the additional dimension of space can be added to the networking "toolbox". However, only a very limited amount of works have addressed this issue. In $[16,17]$ the use of spatial super-channels, which are groups of same-wavelength sub-channels that are transmitted on separate spatial modes but routed together, is proposed. It is highlighted that such super-channels can foster transceiver integration through sharing of a single source laser and a single local oscillator (LO) and that they lighten the DSP load by exploiting information about common-mode impairments (CMIs). In [18] the first hierarchy for dynamic multidimensional spatial and spectral optical networking is proposed and complemented with adaptive coded-modulation to form the basis of a novel elastic networking concept. 


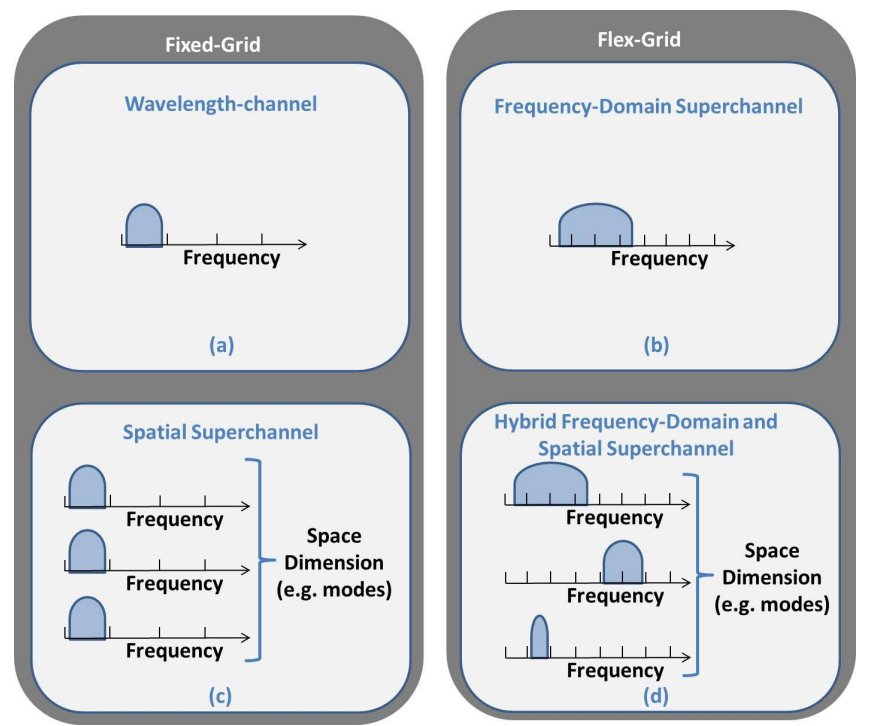

Figure 4 - Introducing flexibility in the frequency and the space domain enables the evolution from conventional wavelength-channels to: frequency domain super-channels, spatial super-channels, and hybrid frequency-domain and spatial super-channels.

Within INSPACE novel network planning techniques are developed that efficiently exploit the additional dimension of space. Spatial super-channels as well as hybrid frequency domain and spatial super-channels will be considered, as shown in Figure 4-(c) and (d) respectively. As conventional RWA and RMLSA algorithms cannot be applied in spatialspectral flexible networks, enhanced routing and resource allocation algorithms are required to be developed. The resources that are now to be assigned to each connection are spectrum and space. "Space" can, for example, correspond to different modes in FMF or cores in MCF. Furthermore, a generic model that can be fine-tuned to capture the capabilities and restrictions posed by the different fibre link types and switching nodes is necessary. The optimization objective of the developed planning algorithms will be tuned to meet: the minimization of capital expenditures for network equipment as well as the maximization of energy efficiency. Furthermore, as the developed algorithms will also be used during the network operation phase, certain restrictions with respect to the maximum allowable computation time need to be considered.

\section{CONTROL PLANE EXTENSIONS REQUIRED FOR SDM NETWORKS}

In existing networks, control layer functionalities can be carried out through either a distributed or a centralized control plane. In distributed networks, there is no specific node with a prominent role; the nodes should exploit a common protocol to exchange information about the network resources and configuration. In a centralized approach the components of the network are under the control of a single element. The latter possess the information related to network configuration, availability, monitored parameters, etc., and then it can perform accurate management of network resources, e.g. by choosing to re-use an already established tunnel, setting up another tunnel between the border routers of the core network, or by performing traffic grooming. A recent architectural framework that is recently gaining a lot of attention from both academic and industrial communities is the so-called Software Defined Networking (SDN) [19]. The principle behind SDN is that the centralized control is separated from network forwarding or the switching plane it is controlling.

SDN seems to be an appropriate control paradigm for the implementation of the control of the reference network architecture envisioned in INSPACE. While current state of the art solutions have only taken into account the flexible spectrum domain, in INSPACE the SDN controller will be properly adapted to deal with the additional "space" domain, thus it can be designed to perform or to request complex operations such as traffic engineering, optimization, and grooming across different physical domains. In particular, if the SDN solution based on OpenFlow protocol (OF) will be eventually considered as the most suitable one for INSPACE, the OF should be extended to provide the above mentioned INSPACE network capabilities. 


\section{REFERENCES}

[1] Peter J. Winzer, "Spatial Multiplexing: The next frontier in network capacity scaling", (Tutorial) in proc. 38th European Conf. on Optical Comm., paper We.1.D.1, London, UK, September 2013.

[2] Jun Sakaguchi et al., "19-core fiber transmission of 19x100x172-Gb/s SDM-WDM-PDM-QPSK signals at 305Tb/", in proc. Optical Fiber Communications Conference, paper PDP5C.1, Los Angeles CA, USA, March 2012.

[3] Takara et al., "1.01-Pb/s (12 SDM/222 WDM/456 Gb/s) Crosstalk-managed Transmission with 91.4-b/s/Hz Aggregate Spectral Efficiency", in proc. 37th European Conf. on Optical Comm., paper PDP Th.3.C.1., Amsterdam, Netherlands, September 2012.

[4] T.Wu, Time Magazine, March 11th, 2010

[5] D.Ellis, J. Zhao, D. Cotter, "Approaching the Non-linear Shannon Limit", Journal of Lightwave Technology, Vol. 28, No. 4, pp $423-433(2010)$

[6] A.D.Ellis, F.C.G.Gunning, "Implementation of Tbit/s Networks", IEEE Photonics Conference 2011, Special Symposium on Terabit Optical Ethernet, paper MW3, (2011)

[7] K. S. Abedin et al., Opt. Ex. 19, 16715 (2011)

[8] S. Randel, OFC 2013, Tutorial OW4F.1

[9] M. Klinkowski and K. Walkowiak, "Routing and Spectrum Assignment in Spectrum Sliced Elastic Optical Path Network", IEEE Communications Letters, vol. 15, no. 8, pp. 884-886, 2011

[10]K. Christodoulopoulos, I. Tomkos, M. Varvarigos, "Elastic Bandwidth Allocation in Flexible OFDM-based Optical Networks", IEEE/OSA Journal of Lightwave Technology, 2011

[11] K. Christodoulopoulos, I. Tomkos, M. Varvarigos, "Dynamic Bandwidth Allocation in Flexible OFDM-based Networks", OFC/NFOEC 2011

[12] E. Palkopoulou et al., "Dynamic Cooperative Spectrum Sharing in Elastic Networks," OFC/NFOEC 2013

[13] F. Cugini et al, "Push-Pull Technique for Defragmentation in Flexible Optical Networks," OFC/NFOEC 2012

[14]Xi Wang et al., "A Hitless Defragmentation Method for Self-optimizing Flexible Grid Optical Networks," ECOC 2012

[15]E. Palkopoulou, et al. "Quantifying Spectrum, Cost, and Energy Efficiency in Fixed-Grid and Flex-Grid Networks," Journal of Optical Communications and Networking (JOCN), Vol. 4 (11), art. no. 6360168 , pp. B42-B51, 2012 (invited)

[16] M. Cvijetic et al., "Dynamic multidimensional optical networking based on spatial and spectral processing," Optics Express 2012

[17] Mark D. Feuer, Lynn E. Nelson, Kazi S. Abedin, Xiang Zhou, Thierry F. Taunay, John F. Fini, Benyuan Zhu, Rejoy Isaac, Roey Harel, Gil Cohen, Dan M. Marom, "ROADM System for Space Division Multiplexing with Spatial Superchannels", OFC/NFOEC 2013, PDP5B.8

[18] M. Feuer et al, "Demonstration of Joint DSP Receivers for Spatial Superchannels," IEEE Photonics Summer Topicals 2012 conference, 2012

[19] N. McKeown, "Software Defined Networking”, INFOCOM 2009. 